\title{
EXPERIMENTS ON THE INTERNET
}

\author{
Chaired by C. Michael Levy, University of Florida, Gainesville
}

\section{Editing a book on the World-Wide Web with 29 collaborators in 8 countries in 12 months: A case in survival tactics}

\author{
C. MICHAEL LEVY \\ University of Florida, Gainesville, Florida \\ and \\ SARAH RANSDELL \\ Florida Atlantic University, Ft. Lauderdale, Florida
}

\begin{abstract}
We describe the techniques that we devised to enable authors who had never met physically to collaborate on a large-scale writing project. The goal was to use the Internet to simulate the close interactions that are possible in a traditional conference to foster cross-fertilization of ideas and to increase the coherence of the book we were writing. We discuss areas that should concern those who consider using the Internet for collaborative work.
\end{abstract}

A year ago we had a long conversation about an important book in our research area, the now-classic Cognitive Processes in Writing, edited by Lee Gregg and Erwin Steinberg (1980). This book grew out of a conference held at Carnegie Mellon University in 1978. Even though a great deal had happened theoretically, methodologically, and empirically in the field of writing in the intervening years, no book had appeared since that captured its breadth and depth of coverage.

We approached many of the original contributors and a number of other active researchers in North America, Europe, the United Kingdom, Israel, and Australia about their interest in contributing to a volume that would showcase some of the latest thinking about the process of writing. The consensus was that the time was ripe. Happily for us, the publisher of the Gregg and Steinberg book was also enthusiastic about our volume. We realized at the outset that we could not possibly recreate the conference that was so much an impetus for the original book. At the same time, our experiences with the Internet led us to wonder how far we could stretch this technology to create something akin to a virtual conference, and to wonder how far we could push the conditions that often govern the development of traditional books. Twelve months later, the book is in the publisher's hands, where final copyediting

Correspondence should be addressed to C. M. Levy, Box 112250, University of Florida, Gainesville, FL 32611-2250 (e-mail: levy@ psych.ufl.edu). is being done. This paper is about some of our experiences over the last year that enabled us to get to this pointexperiences that might help smooth the road to high-tech book editing for those who decide to take this path.

\section{The Prospectus}

We committed to the publisher to produce a book that would exhibit the coherence of volumes that sometimes emerge from conferences and meetings. We suspected that coherence was fostered by the face-to-face interactions that occurred at such meetings and by the opportunities for contributors to read, review, and reflect on the work of others. These interactions and opportunities might result in extensive cross-referencing by authors to each other's chapters, thereby aiding readers to connect ideas across chapters more readily. Because it was not feasible to bring our collaborators together physically, we struggled with the idea of a virtual conference, discovering that we might be able to accomplish our goals if we provided instant availability of chapter drafts on a World-Wide Web (WWW) home page and facilitated frequent professional interactions by using e-mail to broadcast openended questions to the group.

\section{Target Dates and Deliverables}

We assembled potential contributors, virtually speaking, in October of 1994, and announced four major dates on which we needed agreement before the project could begin. We wanted abstracts by the first week of Decem- 
ber; these abstracts would form the critical substance for our publishing prospectus. They would also be a goodfaith gesture of an author's commitment to the project. The next deadline was for a working draft in suitable form for an internal review, due 6 months later. The plan was for us editors to review each chapter, of course, but in order to simulate the give-and-take of a physical conference, we asked each contributor to agree to provide constructive feedback for two chapters that we would assign. Authors had 2 months to write their reviews. Finally, about 3 months after receipt of their internal reviews, we wanted the final revisions in hand to send to the publisher. We were not interested in setting a record for completing a work of this kind. If any record was set, it was because everyone agreed at the outset that this schedule was a reasonable one, given their other commitments.

The Internet symposium at the 1994 SCiP Conference focused on how we could move from being information consumers to becoming information providers by setting up our own home pages. The adventure that we had in developing our book, The Science of Writing, is really all about straddling the line between information provider and information consumer when one uses the Internet for information development. To use the Internet in this way sometimes necessitates violating the spirit of openness of the Internet and dealing with matters such as privacy and security, topics that we address later.

\section{Migration, Transportation, and Communication}

As may become clear, we had only a limited experience base to use in guiding our approach to book development. Using the Internet to simulate a physical conference meant that we needed to give authors as much flexibility as possible in getting their documents to us. Thus, we placed no restrictions on the computer platform or word-processing software used. This decision left us with months of worry about how to deal with the myriad technical migration issues that could result. Fortunately, most of the writing research establishment appears to use either Microsoft Word or Word Perfect on Macintosh or PC platforms. Our Faculty Support Center transferred the chapters composed on Macintosh computers to diskettes that we could read with our PCs. Because Microsoft Word and Word Perfect contain filters that enabled us to read documents that were saved in the format used by many other word processors, this migration concern was, in the end, much ado about nothing.

We needed contributors' documents in electronic form before they could be installed on the WWW server - and later, be typeset. Once again, we gave authors considerable flexibility in ways to transport their documents. This seemed to help maintain their enthusiasm for the project. We dreaded the prospect of receiving hard copy by ordinary mail or fax, because we would then have to scan each page and process it with optical character recognition software. E-mail of plain text documents was slightly more desirable, but this method generally results in the loss of any special formatting that authors have used. Formatting can be retained in documents sent by e-mail, however, if the sender uses a program such as UUENCODE to compress the file and the receiver uses UUDECODE to decompress the file. From our perspective, it was considerably more cumbersome to move documents from the electronic mailbox on our mainframe to their destination on our office computers than it was to move them from a remote system that had file transfer protocol (FTP) software. Fortunately, each of our authors had access to such software, which they could use to send us their text and graphics files. A few preferred to load these files on their own servers and asked us to log on to their machines to copy their files. This was a problem only when the remote system was connected to its Internet node via a very slow modem; in such cases the editor's computer could be tied up for a rather long time.

When security is not an issue, an editor's chores can be dramatically reduced if all authors install their chapters on their own home pages. The authors convert their raw text to hypertext markup language (HTML) files, leaving the editors with only the task of coding the hyperlink from their chapter title in our table of contents to their home page. This was not feasible when we initiated this project because we were the only participants with personal home pages. Moreover, because the process of inserting HTML tags into documents was tedious and errorprone at that time, our authors would have viewed our assigning this burden to them as unreasonable.

Authors needed to be able to communicate frequently and quickly with one another, as well as with us. Using the Internet for this purpose seemed to be ideal. We knew that the Internet was designed to be a highly reliable medium for message transmission. After all, it evolved from a design created as a defense tool to enable communications to inevitably reach their destination even if the network was in tatters as a result of a nuclear holocaust (Levy, 1995). We found that some mail never reached its destination. For example, we had an author in Sweden and an author in Israel whose mail never reached each other when sent directly. Consultants in Sweden and Israel were unable to figure out why. These authors were able to communicate only when they sent their mail to Florida, where it was relayed back to the other side of the Atlantic. Perhaps this breakdown in an apparently direct link resulted from the fact that computer address formats are not globally universal. For example, to send e-mail from one city to another in the same country, the required address might be smith@x.y.z, but to reach the same city from another country, the address sequence may have to be reversed, so that it reads smith@.z.y.x.

\section{Nuts and Bolts}

Once we received a manuscript over the Internet, we migrated it to our favorite word processor. That was easy. It was considerably more difficult to hand code the HTML tags to reflect the relative importance of the author's headings without printed copy indicating author intentions. 
Inserting these tags is critical because without them a document loses all formatting when it is viewed with a graphics browser such as Netscape or Mosaic. A chapter, along with all its tables and figures, becomes one gigantic paragraph printed in monospaced type.

When we started on this project, tools that would insert the appropriate HTML tags automatically were not yet available. The Internet Assistant for Microsoft Word (a template that automatically formats a document with HTML tags) became available for downloading only after the project was half-completed. In the interim, we had to insert all the HTML codes by hand, a process that typically required about $2 \mathrm{~h}$ for a 30 -page chapter. Using the Internet Assistant reduced the formatting time to about $5 \mathrm{~min}$.

Microsoft Word 6.0x and Word Perfect 6.1 $\mathrm{x}$ users can access the sites-www.microsoft.com and www.wp. novell.com-to download self-extracting files that attach to these word processors, enabling the automatic insertion of HTML tags. There are also other commercial products that are aimed at users of other word-processing software, but these are not gratis.

\section{Handling Graphics and Tables}

Currently, WWW browsers support only two formats for graphics files: GIF and JPEG. Many off-the-shelf graphics programs can translate other formats (e.g., bitmaps or Windows metafiles) into the appropriate formats. The minor loss in resolution that sometimes occurs when the translated files are printed is not usually evident when the graphic is rendered by a browser program. In this project, we encouraged authors to use their favorite graphing tool for creating pictorial representations of their models and data, and to send us printed copies, if necessary. Our plan was to scan these prints and directly convert them to the needed formats for those who could not do this themselves.

\section{The Home Page}

After a chapter was marked with HTML tags and references to its graphics links were included, we transferred the files to our WWW server. Our home page simply depicted the table of contents for the book. The book and chapter titles were underscored, indicating that they were hyperlinked to other documents. A click on the book title branched the reader to the prospectus that we had prepared for the publisher. The chapter titles were initially linked to the chapter abstracts, but these were replaced over time with the chapters themselves as they were composed and delivered.

Our plan had been for authors to send us their drafts fairly frequently, and for us to mark up the text with the HTML tags and to notify participants that a new version of Chapter $x$ had been installed on the WWW server. This plan derived from our goal to have an extended virtual conference in which everyone could participate in the evolution of ideas. We displayed drafts of our own chapters on the WWW very early in the process to encourage our colleagues to follow suit. When the review period for chapter drafts began, collaborators could use their browser program to review the entire book and print well-formatted copies of any part on a local printer. They could also save the chapters they were assigned to review on their own computer, use a word processor to insert comments at the appropriate places, and send the resulting document via e-mail to us and the chapter author(s).

\section{Security}

There comes a time during the history of a theoretical idea or empirical finding when the researcher determines that it is ripe for public disclosure. Before that event occurs, researchers carefully pick and choose those with whom they share their ideas. Researchers whose work is in military or leading-edge technology may be more wary than those working in low-tech academic environments, such as writing research labs. Nevertheless, the decision about when the idea is ready for public unveiling is still vested in the researcher. This simple idea is important because it forces researchers to consider the security of information that is distributed over the WWW.

One of the most exciting characteristics of the WWW is its openness. Huge amounts of information are readily accessible to everyone. Web users generally activate a network searching program (such as InfoSeek, The World Wide Web Worm, or Yahoo) to locate the home pages that contain or point to the information being sought. We suspected that few researchers would readily agree to premature public disclosure of their working drafts and anticipated that book publishers would be unhappy with the idea of literally giving away the source materials. It was therefore necessary for us to prevent public access to the book during its development. The technique for accomplishing this goal is very simple, involving the omission of some key information that WWW searching programs expect.

Today's popular search tools compare the search terms with one or both of two components (the header and title tags) in a document file to determine which pages are likely to be good hits. Some tools can go beyond the title and header tag to scan the entire document. If a document does not contain a header tag or a title tag (and a document does not have to have them), users can still use their favorite browser to read the document. If these tags are absent, however, none of the current popular search tools can currently locate the document. It was this limitation of the current search engines that we exploited to keep the work of our collaborators secure from outsiders. As we explained to our collaborators, by not including the header or title tag, we did the equivalent of building an igloo in the Alaskan tundra. The taxing authorities didn't know we were even there, but our colleagues could find us anytime. Using our complete WWW address, they had the latitude and longitude coordinates. Search tools are almost certain to become more sophisticated, and before too long they may be capable of locating appropriate documents that contain neither a title nor a header tag. This means that others hoping to use the Internet in a similar way will have to deal with the security 
of their documents differently. One approach to consider is that of protecting access to the document with a form that collects a password from the reader, and sharing the password only with collaborators. If the document is a book, and chapters are stored as separate HTML files, each chapter would also have to be protected by a password.

\section{The Writing Process}

We saw this project as an opportunity to study the writing process itself, as well as to produce a book about writing. We had assembled a group of professional writers writing about their writing research over an extended period of time. It could produce a writing researcher's dream database. If our collaborators updated their chapter drafts on a fairly regular basis, and shared these revisions as they were produced, at the end of the project, we would have a rich corpus of writing protocols that might form the basis of several future PhD theses. But despite our attempts at being catalysts, putting our earliest, roughest drafts on the WWW home page, our collaborators wouldn't participate in this way. They submitted drafts only when they had to.

It may be the case that researchers in the area of writing are particularly self-conscious about how they compose. Perhaps this results in a reticence about sharing their prose before they are satisfied that it is as well crafted as they want it to be. Or it could be that writers have a reluctance to "share" revision processes. Our own research (Levy \& Ransdell, 1995) suggests that revising is considerably more effortful than planning or generating text, particularly the first time around.

In summary, using the WWW allowed us to create a collaborative project in the form of 19 chapters from 29 authors, many of whom had never physically met, in what may be record time for this type of work. Thus, part of our story is that it can be done. The capability of a team of collaborators to keep abreast of frequent changes to a project and to offer constructive advice to one another quickly and efficiently are characteristics of this effort that it may be feasible to incorporate into group projects in other contexts. The other part of the story is that virtually, webfacilitated revising and reviewing of collaborators' ongoing work clearly does not replace face-to-face conferencing. This initial effort suggests, though, that such interactions may help editors make their books more cohesive.

\section{REFERENCES}

GregG, L. W., \& STEINBerg, E. R. (1980). Cognitive processes in writing. Hillsdale, NJ: Erlbaum.

LEVY, C. M. (1995). Mosaic and the information superhighway: A virtual tiger in your tank. Behavior Research Methods, Instruments, \& Computers, 27, 187-192.

LEVY, C. M., \& RANSDELL, S. (1995). Is writing as difficult as it seems? Memory \& Cognition, 23, 767-779.

(Manuscript received November 27, 1995; revision accepted for publication January 30,1996 .) 